\title{
Neonatal COVID-19 exposures and infections: a systematic review of modes of transmission, manifestations and management
}

Obumneme B. Ezeanosike', Dorathy C. Obu', Olapeju W. Daniyan', Onyinye U. Anyanwu', ljeoma N Okedo-Alex², Ifeyinwa C Akamike², Edak Ezeanosike ${ }^{3}$

1 Department of Paediatrics, Alex Ekwueme Federal University Teaching Hospital, Abakaliki, Nigeria, ${ }^{2}$ Department of Community Medicine, Alex Ekwueme Federal University Teaching Hospital, Abakaliki, Nigeria, ${ }^{3}$ Department of Ophthalmology, Alex Ekwueme Federal University Teaching Hospital, Abakaliki, Nigeria

Keywords: neonate, sars-cov-2, covid-19

https://doi.org/10.29392/001c.16684

\section{Journal of Global Health Reports}

Vol. 4, 2020

\section{Background}

Since the emergence of the novel coronavirus disease (COVID-19) in December 2019, several publications have emerged describing the manifestations of the disease in different age groups. Reports from the newborn group have however been sparse. This review summarizes the mode of transmission, clinical presentations as well as treatment and outcomes of COVID-19 in neonates.

\section{Methods}

A systematic search for literature was conducted in April and in August 2020. The databases searched include PUBMED, Ovid MEDLINE, EMBASE, CINAHL, Web of Science, and Google Scholar. The search terms used included COVID-19, SARS-CoV-2, Corona virus, Newborn, Neonate, exposure, vertical transmission, breastfeeding, droplet infection. We included primary studies that were carried out globally either in hospitals or in the communities such as case reports, case series, cross-sectional studies and other qualitative or quantitative studies and published in English language, between January and August 2020.

\section{Results}

Fifty six studies were included in this review. Studies were conducted in China, Iran, South Korea, Peru, USA, Spain, Belgium, France, and Australia. A total of 416 neonates were examined between few hours old and 28 days of age. A total of 38 neonates had PCR-confirmed COVID-19 disease following testing. Fourteen (36.8\%) out of the 38 neonates that tested positive had no symptoms. Clinical features reported were mild and include systemic features such as fever (4.1\%), respiratory features such as tachypnoea (5.1\%), cardiovascular, gastrointestinal, and dermatological features. Radiological features reported include pneumonia (1\%), ground glass opacity (2.9\%), consolidation $(0.7 \%)$, increased lung markings $(0.3 \%)$. Person to person transmission was more common. Treatment was generally supportive.

\section{Conclusions}

There is little evidence of vertical transmission in neonates. Neonatal survival following COVID-19 infection is very high perhaps due to mild nature of the disease in this age group. More research using higher quality study designs and methodologies is recommended.

A novel coronavirus disease 2019 (COVID-19) caused by severe acute respiratory syndrome coronavirus 2 (SARSCoV-2) emerged in December 2019 in Wuhan, Hubei Province, China. ${ }^{1}$ Since then the disease has spread to many countries and was declared a public health emergency of international concern (PHEIC) by the World health organization on 30 January $2020 .^{2}$ Prior to this time, 6 coronavirus species were known to cause human disease, four of which is known to cause common cold-like infections in immunecompetent humans (229E,OC43,HKU1,NL63), the other two namely, severe acute respiratory syndrome coronavirus(SARS-COV) and the Middle East respiratory syndrome coronavirus (MERS-CoV), are zoonotic in origin and are known to cause severe respiratory disease in humans. A lot is known about the disease whose common symptoms are fever, cough and shortness of breath. Globally, as at $8^{\text {th }}$ 
September 2020, there have been 27,236,916 confirmed cases of COVID-19, including 891,031 deaths, reported to WHO with increasing numbers everyday. ${ }^{3}$

In spite of all efforts to contain the pandemic, the number of infections and deaths from COVID-19 keeps increasing. Elderly persons and adults with other background medical conditions have been the worst affected. ${ }^{4}$ Many documented studies on COVID-19 are mostly on adults and some on older children with few on neonates.

In Italy, Japan, and Thailand, there were community based infections with cases of SARS-CoV-2 associated pneumonia reported in children. ${ }^{4-6}$ Reported paediatric cases were mainly family cluster cases with epidemiological links to adults. ${ }^{4-6}$ Peadiatric cases appear to have a milder clinical course than those in adults and rarely deaths have been reported in children. ${ }^{6}$ These may imply that pediatric cases may play a role in community spread of SARS-CoV-2 since the viral shedding may continue from nasal secretions as well as through fecal matter for several weeks after diagnosis. ${ }^{7}$ Infections of SARS-CoV-2 in neonates have been said to be mild also. ${ }^{7}$ The reason behind the less susceptibility and mild infections of SARS-CoV-2 in neonates is largely unclear especially as neonates are not generally protected from respiratory viral infections. Although vertical transmission of COVID-19 is still being debated, maternal COVID19 infections especially when associated with severe hypoxia and fever may result in fetal distress, premature delivery and other risks.

Limited evidence exists however on mode of transmission, prevalence and clinical features of COVID-19 in neonates. It has been shown that mothers may transmit the infections by droplets during breastfeeding or by vertical transmission. ${ }^{8}$ Common presentations of fever and respiratory signs have been found to occur in neonates with COVID-19 however other existing co- morbidities such as prematurity and chromosomal disorder makes it unclear as to the actual cause of these signs. ${ }^{1,9}$ Other times, neonates may present with non-specific symptoms such as lethargy and dehydration making diagnosis difficult. Treatment of COVID-19 have been largely symptomatic with no definitive therapy. ${ }^{3,7}$ Drug trials have been largely done in adult population. A clear understanding of the symptomatology of COVID-19 among neonates, its mode of transmission and diagnosis is needed to improve public health and clinical response. There is some knowledge gap with regards to COVID-19 in neonates despite its epidemiological importance, and therefore, it is our objective to describe the documented modes of transmission, clinical features, treatment, complications and outcome of infections of SARS-CoV-2 in neonates by a systematic review of existing literature.

\section{METHODS}

The Preferred Reporting Items for systematic Reviews and Meta-Analysis (PRISMA) checklist for reporting a systematic review or meta-analysis protocol was used for this review (Online Supplementary Document, Appendix 1). ${ }^{10}$

\section{SEARCH STRATEGY AND SELECTION CRITERIA}

A systematic search for literature was conducted on $5^{\text {th }}$ April 2020 and on $4^{\text {th }}$ August 2020. The databases searched include PubMed, Ovid MEDLINE, EMBASE, CINAHL, Web of Science, and Google Scholar. Other COVID-19 data provided freely by various institutions on their websites were also retrieved. The search terms used included keywords, text words and medical subject headings (MeSH) terms and subheadings of the following: COVID-19, SARS-CoV-2, Corona virus, Newborn, Neonate, exposure, vertical transmission, breastfeeding, droplet infection.

\section{Box 1. Selection criteria}

\section{Inclusion criteria}

- Primary Studies (Original studies) that used any study design

- Studies published in English language

- Studies that reported COVID-19 exposure, transmission, infection, and disease in the newborn

Exclusion criteria

- Review articles

- Studies published in other languages

\section{INCLUSION AND EXCLUSION CRITERIA}

We included primary studies that were carried out globally either in hospitals or in the communities such as case reports, case series, cross-sectional studies and other qualitative or quantitative studies and published in English language, between January and August 2020 which reported COVID-19 exposure, transmission, infection, and disease in the newborn.. Review articles and studies in other languages apart from English were excluded.

\section{STUDY SELECTION}

A total 1250 studies were identified from the initial search of databases and institutional sources 1830 from other sources including institutionally provided data. After duplicates were removed, the number of remaining publications was 530 articles, and these were reviewed for inclusion based on information contained in titles and abstracts.

Studies not addressing exposure of transmission to or infection in newborns were excluded to give a total of 109 full text articles. These were assessed further, and 56 full text articles were included for the review (Figure 1).

\section{DATA EXTRACTION}

A data extraction form was developed and reviewed by all authors. Data was extracted for each paper using the standardised form with the following domains: the name of first 


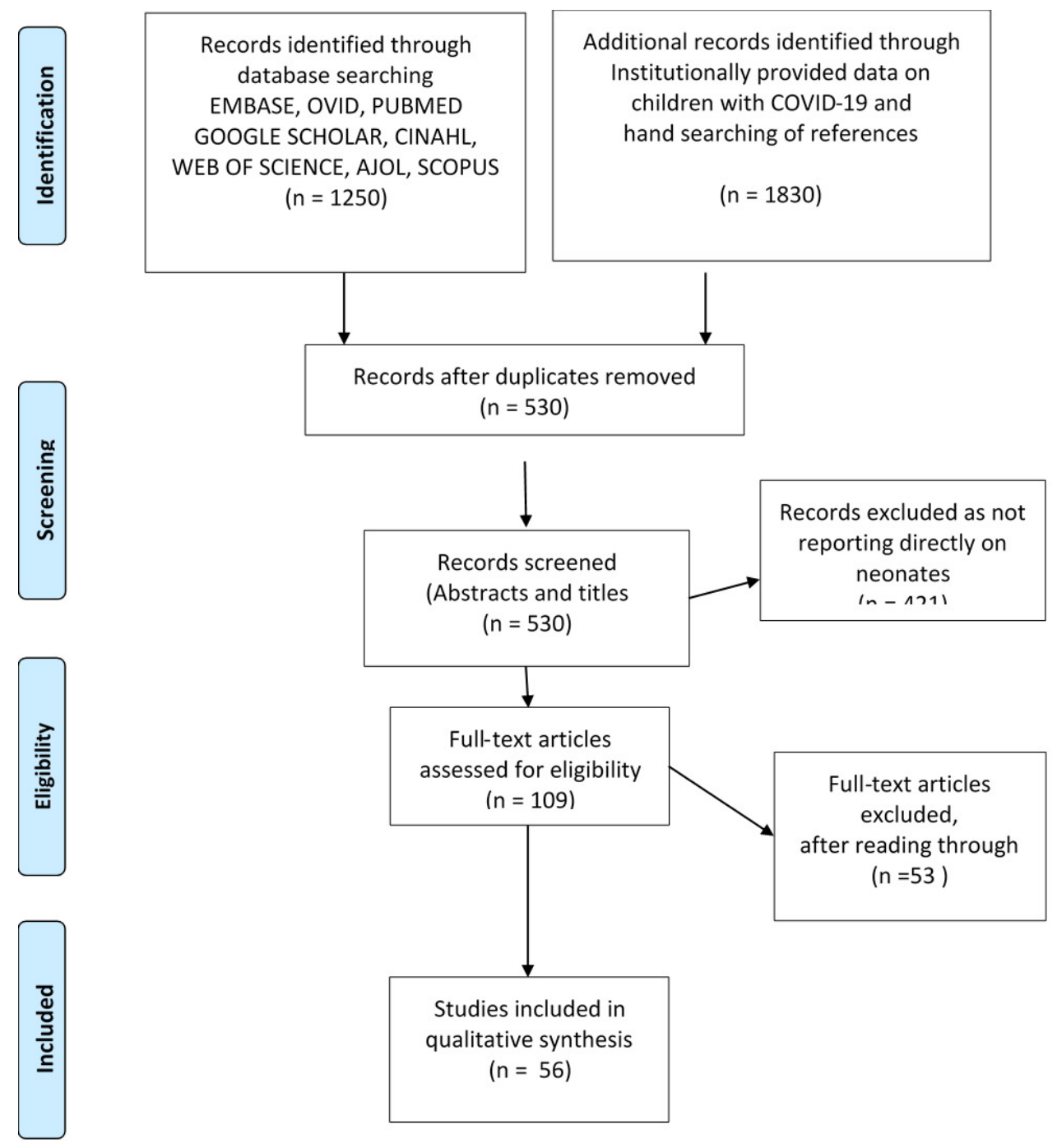

Figure 1. PRISMA FLOW DIAGRAM

author and year of publication, study location, study design, clinical features, laboratory and radiological findings, diagnosis of Covid-19, treatment and outcome. Two of the reviewers extracted the data independently and discrepancies were resolved by discussion and consensus. The references were tracked using EndNote reference manager where duplicates were also removed.

\section{QUALITY ASSESSMENT OF STUDIES}

Quality assessment of studies was done using the Newcastle Ottawa Scale for Case Control Studies, ${ }^{11}$ Quality Assessment Tool for Observational Cohort and Cross-Sectional Studies, ${ }^{12}$ and the Tool for Evaluating the Methodological Quality of Case Reports and Case Series ${ }^{13}$ (Online Supplementary Document, Appendix 2). All the case reports and case series included in this review fulfilled the quality criteria (Online Supplementary Document, Appendix 3). For the cross sectional studies, most of the studies had "not applicable" or "cannot determine" as responses to the questions on sample size calculation, measurement of exposure and outcome variables and on statistical analysis, but fulfilled the criteria with regards to questions on study objec- tives, study population and sampling (Online Supplementary Document, Appendix 4).

\section{ETHICS CONSIDERATIONS}

Ethical approval was not required for this study because it is a systematic review with no direct involvement of human or animal study participants.

\section{RESULTS}

\section{STUDY CHARACTERISTICS}

Fifty-six studies were included in this review. Thirty one of the studies were conducted in China while the remaining studies were carried out in Iran (five) $)^{1,14-17}$, South Korea (two $^{2,18}$, USA (seven $)^{3,19-24}$, Italy (four) ${ }^{25-28}$, Spain (three) $^{29-31}$, Peru (one $)^{4}$, France (one) ${ }^{32}$, Australia (one) ${ }^{33}$, Belgium (one) ${ }^{34}$ (Table 1). All the studies were published between January and August 2020 (Table 1). The study designs included in this review were 33 case reports, 13 case series, one expanded case series, one case control study, 
and eight cross-sectional studies(Table 1). From the included studies, a total of 416 neonates were examined between few hours old and 28 days of age. A total of 38 neonates had PCR-confirmed COVID-19 disease following testing (Table 2). Fourteen (36.8\%) out of the 38 neonates that tested positive had no symptoms. ${ }^{14-16,23,25,29,34-39}$ 
Table 1: General characteristics of studies included in the review

\begin{tabular}{|c|c|c|c|c|c|c|}
\hline First Author & Year & Month & Country & $\begin{array}{l}\text { Region or } \\
\text { State }\end{array}$ & Type of Study & $\begin{array}{l}\text { No of } \\
\text { neonates }\end{array}$ \\
\hline Alzamora 4 & 2020 & April & Peru & NS & Case report & 1 \\
\hline Dehan Liu 40 & 2020 & March & China & Wuhan & Case series & 11 \\
\hline Mi Seon $\mathrm{Han}^{2}$ & 2020 & April & $\begin{array}{l}\text { South } \\
\text { Korea }\end{array}$ & Seoul & Case report & 1 \\
\hline Peng $Z^{5}$ & 2020 & April & China & Chongqing & Case report & 1 \\
\hline $\mathrm{Na} \mathrm{Li}{ }^{41}$ & 2020 & March & China & Wuhan & case control & 17 \\
\hline Pu Yang 42 & 2020 & April & China & Wuhan & Case Series & 7 \\
\hline Siyu Chen ${ }^{9}$ & 2020 & March & China & Hubei & Case series & 5 \\
\hline Wei Liư33 & 2020 & April & China & Wuhan & Cross sectional & 19 \\
\hline Xiali Xiong 6 & 2020 & April & China & Beijing & Case report & 1 \\
\hline Lingkong Zeng 44 & 2020 & March & China & Wuhan & Cross sectional & 33 \\
\hline Kamali Aghdam ${ }^{1}$ & 2020 & April & Iran & Tehran & Case report & 1 \\
\hline Lingkong Zeng7 & 2020 & April & China & Wuhan & Case report & 1 \\
\hline Wang Shaoshuai ${ }^{8}$ & 2020 & March & China & Wuhan & Case report & 1 \\
\hline Chen Yan 45 & 2020 & March & China & Wuhan & Case report & 4 \\
\hline Munoz ${ }^{3}$ & 2020 & May & USA & Houston & Case report & 1 \\
\hline Zhu ${ }^{46}$ & 2020 & Feb" & China & Wuhan & Cross sectional & 10 \\
\hline Nan Yu 47 & 2020 & March & China & Wuhan & Cross sectional & 7 \\
\hline Cuifang Fan 48 & 2020 & March & China & Wuhan & Case series & 2 \\
\hline $\mathrm{Li} \mathrm{Yao} 49$ & 2020 & March & China & Hefei & Case report & 1 \\
\hline Zhuang Siying 50 & 2020 & February & China & Wuhan & Case report & 1 \\
\hline Bai Bailiang 51 & 2020 & March & China & Shaanxi & Case report & 1 \\
\hline Patek Paul 21 & 2020 & April & USA & Royal oak & Case report & 1 \\
\hline Sinelli $\mathrm{MT}^{27}$ & 2020 & May & Italy & Monza & Case report & 1 \\
\hline $\begin{array}{l}\text { Hantoushzadeh } \\
\text { Sedigheh }^{14}\end{array}$ & 2020 & April & Iran & Tehran & Case series & 7 \\
\hline Vikramaditya Dumpa 22 & 2020 & May & USA & New York & Case Report & 1 \\
\hline Xiaolin $\mathrm{Hu}^{37}$ & 2020 & April & China & Wuhan & Case series & 7 \\
\hline Alonso Diaz 31 & 2020 & April & Spain & Madrid & Case report & 1 \\
\hline Iqbal S24 & 2020 & April & USA & Washington & Case report & 1 \\
\hline Khan $S^{52}$ & 2020 & April & China & Wuhan & Case series & 17 \\
\hline Belinda Lowe 33 & 2020 & April & Australia & Queensland & Case report & 1 \\
\hline Ferrazzi $E^{28}$ & 2020 & April & Italy & Lombardia & Cross sectional & 42 \\
\hline Zamaniyan $\mathrm{M}^{15}$ & 2020 & April & Iran & Sari & Case report & 1 \\
\hline Lu D 53 & 2020 & April & China & Hefei & Case report & 1 \\
\hline Wang J54 & 2020 & March & China & Wuhan & Case report & 1 \\
\hline Sun $M^{38}$ & 2020 & May & China & Zhengzhou & Case series & 3 \\
\hline Wu Y55 & 2020 & May & China & Wuhan & Case Series & 5 \\
\hline Piersigilli34 & 2020 & May & Belgium & Brussels & Case report & 1 \\
\hline Chen Huijun 39 & 2020 & February & China & Wuhan & Cross sectional & 9 \\
\hline Xiaotong Wang 56 & 2020 & February & China & Suzhou & Case report & 1 \\
\hline Zhi-Jiang Zhang 36 & $/ 2020$ & March & China & & Case series & 4 \\
\hline Zeng $\mathrm{H}^{57}$ & 2020 & March & China & Wuhan & Case series & 6 \\
\hline Dong Lan 58 & 2020 & March & China & Wuhan & Case report & 1 \\
\hline Buonsenso 25 & 2020 & April & Italy & $?$ & Case series & 2 \\
\hline Noelle 23 & 2020 & May & USA & New York & Cross sectional & 18 \\
\hline Jie $\operatorname{Yan}^{59}$ & 2020 & July & China & Wuhan & Expanded case & 116 \\
\hline
\end{tabular}




\begin{tabular}{|c|c|c|c|c|c|c|}
\hline First Author & Year & Month & Country & $\begin{array}{l}\text { Region or } \\
\text { State }\end{array}$ & Type of Study & $\begin{array}{l}\text { No of } \\
\text { neonates }\end{array}$ \\
\hline & & & & & series & \\
\hline Yan Ting wu 35 & 2020 & July & China & Wuhan & Cross sectional & 30 \\
\hline Eun-Kyung Lee ${ }^{18}$ & 2020 & July & $\begin{array}{l}\text { South } \\
\text { Korea }\end{array}$ & Daegu & Case report & 1 \\
\hline Fatemeh Eghbalian 16 & 2020 & July & Iran & Hamadan & Case report & 1 \\
\hline Gregorio-Hernández 29 & 2020 & June & Spain & $?$ & Case series & 2 \\
\hline Serafina Perrone 26 & 2020 & May & Italy & Parma & Case report & 1 \\
\hline Mahdavi ${ }^{17}$ & 2020 & June & Iran & Shahroud & Case Report & 1 \\
\hline Rishi Lumba ${ }^{19}$ & 2020 & July & USA & New York & Case report & 1 \\
\hline Rocio 30 & 2020 & June & Spain & Barcelona & Case report & 1 \\
\hline Salik 20 & 2020 & May & USA & New York & Case report & 1 \\
\hline Ying Xiong60 & 2020 & July & China & Wuhan & Case report & 1 \\
\hline Soumeth Abasse 32 & 2020 & August & France & Mayotte & Case report & 1 \\
\hline
\end{tabular}


Table 2: Clinical, laboratory presentations, and management outcomes of COVID-19 exposed and

infected neonates

\begin{tabular}{|c|c|c|c|c|c|c|}
\hline First Author & Clinical features & Laboratory findings & Radiological findings & Diagnosis of COVID-19 & Treatment & Outcome \\
\hline LiYao & None & Normal & ND & $\begin{array}{l}\text { Negative nasopharyngeal, anal, } \\
\text { peripheral blood samples }\end{array}$ & None & Alive \\
\hline Bai Bailiang. & None & ND & ND & $\begin{array}{l}\text { Negative amniotic, placental, breast } \\
\text { milk and cord blood sample }\end{array}$ & Supportive & Alive \\
\hline Patek Paul & $\begin{array}{l}\text { Fever, cyanosis, reduced milk } \\
\text { intake, fussiness }\end{array}$ & $\begin{array}{l}\text { Decreased neutrophil, deranged liver } \\
\text { function test }\end{array}$ & $\begin{array}{l}\text { bilateral perihilar streaking } \\
\text { without focal }\end{array}$ & Positive nasopharyngeal sample & $\begin{array}{l}\text { Supportive, } \\
\text { oxygen, } \\
\text { antibiotic, } \\
\text { antiviral }\end{array}$ & Alive \\
\hline Sinelli MT & $\begin{array}{l}\text { Cyanosis, poor suck, } \\
\text { bradypnoea }\end{array}$ & $\begin{array}{l}\text { Normal white blood cell parameters, } \\
\text { Arterial blood gas analysis } \\
\text { demonstrated moderate hypoxia }\end{array}$ & $\begin{array}{l}\text { mild bilateral ground-glass } \\
\text { opacities }\end{array}$ & Positive nasopharyngeal sample & $\begin{array}{l}\text { Supportive, } \\
\text { oxygen, } \\
\text { antibiotic }\end{array}$ & Alive \\
\hline $\begin{array}{l}\text { Hantoushzadeh } \\
\text { Sedigheh }\end{array}$ & None (5) Not applicable (5) & $\begin{array}{l}\text { ND (4) Lymphopenia (1) Not applicable } \\
\text { (5) }\end{array}$ & ND (5) Not applicable (3) & $\begin{array}{l}\text { Negative (2) Positive on day } 7 \text { (1) ND (3) } \\
\text { Not applicable (5) }\end{array}$ & $\begin{array}{l}\text { Supportive } \\
\text { (2) None } \\
\text { (3) Not } \\
\text { applicable } \\
\text { (5) }\end{array}$ & $\begin{array}{l}\text { Fetal } \\
\text { demise } \\
\text { (3) Alive } \\
\text { (5) }\end{array}$ \\
\hline $\begin{array}{l}\text { Vikramaditya } \\
\text { Dumpa }\end{array}$ & $\begin{array}{l}\text { Fever, feed intolerance, } \\
\text { tachycardia }\end{array}$ & Normal white blood cell parameters & ND & Positive nasopharyngeal sample & $\begin{array}{l}\text { Supportive, } \\
\text { antibiotic }\end{array}$ & Alive \\
\hline Xiaolin Hu & None (7) & $\begin{array}{l}\text { Normal white blood cell parameters } \\
\text { (7), normal liver function test (7), } \\
\text { normal creatinine kinase (7), normal C- } \\
\text { reactive protein (7), normal } \\
\text { procalcitonin (7) }\end{array}$ & Normal (7) & $\begin{array}{l}\text { Positive nasopharyngeal, oropharngeal, } \\
\text { amniotic fluid, peripheral blood sample } \\
\text { (1) Others (6) negative samples }\end{array}$ & $\begin{array}{l}\text { Supportive } \\
\text { (1), } \\
\text { antibiotic } \\
\text { (1), None } \\
\text { (6) }\end{array}$ & Alive (7) \\
\hline Alonso Díaz & $\begin{array}{l}\text { Fast breathing, dyspnea, } \\
\text { cyanosis, }\end{array}$ & Mild acidosis & $\begin{array}{l}\text { Ground glass opacities } \\
\text { mainly in the perihilar area }\end{array}$ & Positive nasopharyngeal sample & $\begin{array}{l}\text { Supportive, } \\
\text { oxygen, } \\
\text { antibiotic }\end{array}$ & Alive \\
\hline Chen S & None (5) & ND (5) & ND (5) & Negative nasopharyngeal sample & $\begin{array}{l}\text { Supportive } \\
\text { (5) } \\
\text { Antibiotic } \\
\text { (5) } \\
\text { Antiviral } \\
\text { (5) }\end{array}$ & All alive \\
\hline lqbal S & None & Normal white blood cell parameter & ND & $\begin{array}{l}\text { Negative nasopharyngeal, } \\
\text { oropharyngeal, rectal sample }\end{array}$ & Supportive & Alive \\
\hline Chen Y & $\begin{array}{l}\text { None (2) Fast breathing (1) } \\
\text { Dyspnea (1) Edema (2) Rash } \\
\text { (20 }\end{array}$ & $\begin{array}{l}\text { Normal white blood cell parameters (3) } \\
\text { Normal LFT (3) ND (1) }\end{array}$ & Normal (3) ND (1) & Negative throat sample (4) & $\begin{array}{l}\text { Supportive } \\
\text { (4) Oxygen } \\
\text { (1) }\end{array}$ & Alive \\
\hline Xiong $\mathrm{X}$ & None & Normal immunoglobin & ND & $\begin{array}{l}\text { Negative nasopharyngeal, throat, rectal, } \\
\text { amniotic fluid, placental, breast milk }\end{array}$ & Supportive & Alive \\
\hline Khan S & Not available & ND & Normal USS finding & $\begin{array}{l}\text { Positive throat samples in } 2 \text { babies, } \\
\text { Negative amniotic, placental, cord blood } \\
\text { in } 17 \text { babies }\end{array}$ & Supportive & Alive \\
\hline Belinda Lowe & None & ND & ND & Negative throat sample & Supportive & Alive \\
\hline Ferrazzi E & Abdominal distension, fast & ND & ND & Positive nasopharyngeal samples (3) & Supportive & All alive \\
\hline
\end{tabular}




\begin{tabular}{|c|c|c|c|c|c|c|}
\hline First Author & Clinical features & Laboratory findings & Radiological findings & Diagnosis of COVID-19 & Treatment & Outcome \\
\hline & $\begin{array}{l}\text { breathing, dyspnea, cyanosis, } \\
\text { feed intolerance, reduced milk } \\
\text { intake (1) }\end{array}$ & & & & $\begin{array}{l}(42) \\
\text { Oxygen (1) } \\
\text { Antibiotic } \\
\text { (1) }\end{array}$ & \\
\hline Zamaniyan M & None & ND & ND & $\begin{array}{l}\text { Positive nasopharyngel and amniotic } \\
\text { fluid samples. Negative throat sample }\end{array}$ & Supportive & Alive \\
\hline Lu D & None & ND & Normal findings & $\begin{array}{l}\text { Negative nasopharyngeal, } \\
\text { oropharyngeal, peripheral blood }\end{array}$ & Supportive & Alive \\
\hline Munoz AC & $\begin{array}{l}\text { Nasal congestion, fast } \\
\text { breathing, dyspnea, reduced } \\
\text { milk intake, tachycardia, } \\
\text { hypothermia, hypotension }\end{array}$ & $\begin{array}{l}\text { Lymphocytosis, increased cytokine } \\
\text { levels, acidosis }\end{array}$ & $\begin{array}{l}\text { bilateral linear opacities and } \\
\text { consolidation in right upper } \\
\text { lobe }\end{array}$ & Positive nasopharyngeal sample & $\begin{array}{l}\text { Supportive, } \\
\text { oxygen, } \\
\text { antibiotic }\end{array}$ & Alive \\
\hline Wang J & $\begin{array}{l}\text { Fever, vomiting, reduced milk } \\
\text { intake, diarrhea }\end{array}$ & $\begin{array}{l}\text { Thrombocytopenia, positive for occult } \\
\text { blood, Normal LFT, CK, } \\
\text { immunoglobulin, cytokines }\end{array}$ & Patchy fuzzy shadows & Positive anal, throat sample & $\begin{array}{l}\text { Supportive, } \\
\text { interferon }\end{array}$ & Alive \\
\hline Sun $M$ & $\begin{array}{l}\text { None (1) Fast breathing (2) } \\
\text { Dyspneoa (2) }\end{array}$ & Neutrophilia (1) & Normal finding (1), ND (2) & Positive (1) Negative (2) & $\begin{array}{l}\text { Supportive } \\
\text { (3) Oxygen } \\
\text { (2) } \\
\text { Antibiotic } \\
\text { (2) }\end{array}$ & All alive \\
\hline WuY & None (5) & $\begin{array}{l}\text { Leucopenia (1) Lymphopenia (1) } \\
\text { Positive immunoglobulin (1) }\end{array}$ & ND (1) & $\begin{array}{l}\text { Negative throat sample (1) Positive } \\
\text { breast milk sample (1) }\end{array}$ & $\begin{array}{l}\text { Supportive } \\
\text { (5) }\end{array}$ & All alive \\
\hline Wei Liu & None & $\begin{array}{l}\text { Normal white blood cell, CK, LFT, } \\
\text { immunoglobulin, cytokine }\end{array}$ & Normal & $\begin{array}{l}\text { Negative stool, throat, amniotic fluid, } \\
\text { breast milk samples }\end{array}$ & Supportive & Alive \\
\hline Kamali Aghdam & $\begin{array}{l}\text { Fever, fast breathing, dyspnea, } \\
\text { lethargy, tachycardia, mottling }\end{array}$ & $\begin{array}{l}\text { Normal white blood cell parameters, } \\
\text { LFT, CK }\end{array}$ & Normal & Positive nasopharyngeal sample & $\begin{array}{l}\text { Supportive, } \\
\text { oxygen, } \\
\text { antibiotic, } \\
\text { antiviral }\end{array}$ & Alive \\
\hline Piersigilli & None & Lymphopenia & Bilateral streaky infiltrates & $\begin{array}{l}\text { Positive nasopharyngeal sample, } \\
\text { Negative breast milk sample }\end{array}$ & $\begin{array}{l}\text { Supportive, } \\
\text { oxygen }\end{array}$ & Alive \\
\hline Zhu Huaping & $\begin{array}{l}\text { Fever (), abdominal distension } \\
\text { (), fast breathing (6), cyanosis, } \\
\text { feeding intolerance, vomiting, } \\
\text { reduced milk intake, } \\
\text { tachycardia }\end{array}$ & $\begin{array}{l}\text { Thrombocytopenia (2), deranged LFT } \\
\text { (2), }\end{array}$ & $\begin{array}{l}\text { Evidence of Infections, RDS, } \\
\text { Pneumothorax, granular and } \\
\text { scattered patchy shadows }\end{array}$ & Negative nasopharyngeal sample & $\begin{array}{l}\text { Supportive, } \\
\text { oxygen, }\end{array}$ & $\begin{array}{l}\text { Alive (9) } \\
\text { Neonatal } \\
\text { death (1) }\end{array}$ \\
\hline Peng Z & Fast breathing, moaning & Leucopenia, reduced procalcitonin & Reduced lung volume & $\begin{array}{l}\text { Negative saliva, plasma, urine, anal, } \\
\text { throat sample }\end{array}$ & $\begin{array}{l}\text { Supportive, } \\
\text { oxygen, } \\
\text { antibiotics }\end{array}$ & Alive \\
\hline Cuifang Fan & $\begin{array}{l}\text { Fever (1), abdominal } \\
\text { distension (1) }\end{array}$ & Lymphopenia (2) & Diffuse haziness (1) ND (1) & $\begin{array}{l}\text { Negative nasopharyngeal, amniotic } \\
\text { fluid, placental, breast milk, cord blood } \\
\text { samples }\end{array}$ & $\begin{array}{l}\text { Supportive, } \\
\text { antibiotics }\end{array}$ & Alive \\
\hline Yu Nan & None (6) Fast breathing (1) & ND (7) & $\begin{array}{l}\text { Evidence of mild pulmonary } \\
\text { infection (1) }\end{array}$ & $\begin{array}{l}\text { Positive nasopharyngeal sample (1) } \\
\text { Negative nasopharyngeal sample (2) } \\
\text { ND (4) }\end{array}$ & $\begin{array}{l}\text { Supportive } \\
\text { (1) } \\
\text { Antibiotic } \\
\text { (1) }\end{array}$ & All alive \\
\hline Zeng Lingkong & Fever (2), fast breathing (3), & Leucocytosis (1), normal LFT (3), & Evidence of pneumonia (3) & Positive nasopharyngeal and anal & Supportive & All alive \\
\hline
\end{tabular}




\begin{tabular}{|c|c|c|c|c|c|c|}
\hline First Author & Clinical features & Laboratory findings & Radiological findings & Diagnosis of COVID-19 & Treatment & Outcome \\
\hline & lethargy (1), vomiting (1) & increased CK (1) & & $\begin{array}{l}\text { samples (3) Negative amniotic fluid, } \\
\text { breast milk and cord blood samples (3) }\end{array}$ & $\begin{array}{l}\text { (3), Oxygen } \\
\text { (1) } \\
\text { antibiotic } \\
\text { (1) }\end{array}$ & \\
\hline Huijun Chen & ND (9) & Increased CK (1) & ND (9) & $\begin{array}{l}\text { Positive nasopharyngeal (1), negative } \\
\text { nasopharyngeal samples (5), ND (3) } \\
\text { Negative amniotic, breast milk and cord } \\
\text { blood samples (9) }\end{array}$ & $\begin{array}{l}\text { Supportive } \\
\text { (9) }\end{array}$ & All alive \\
\hline Xiaotong Wang & None & ND & ND & $\begin{array}{l}\text { Negative stool, throat, amniotic fluid, } \\
\text { placental and cord blood samples }\end{array}$ & Supportive & Alive \\
\hline $\begin{array}{l}\text { Zhi-Jiang } \\
\text { Zhang }\end{array}$ & $\begin{array}{l}\text { None (1) Fever (2), dyspnea } \\
\text { (1), vomiting (1), cough (1) }\end{array}$ & ND (4) & $\begin{array}{l}\mathrm{ND}(1) \text { Increased lung } \\
\text { markings }\end{array}$ & $\begin{array}{l}\text { Positive nasopharyngeal samples ( } 2 \text { ) } \\
\text { Positive anal samples ( } 2 \text { ) }\end{array}$ & $\begin{array}{l}\text { Supportive } \\
\text { (4) }\end{array}$ & All alive \\
\hline Zeng H & None & Elevated immunoglobulin (2) & ND & $\begin{array}{l}\text { Negative throat and peripheral blood } \\
\text { samples }\end{array}$ & $\begin{array}{l}\text { Not } \\
\text { available }\end{array}$ & All alive \\
\hline Dong Lan & None & $\begin{array}{l}\text { Leukocytosis, elevated } \\
\text { immunoglobulin and cytokines, }\end{array}$ & Normal & Negative nasopharyngeal samples & $\begin{array}{l}\text { Not } \\
\text { available }\end{array}$ & Alive \\
\hline Zeng Lingkong & $\begin{array}{l}\text { Ruddy skin appearance, fast } \\
\text { breathing, vomiting, sneezing, } \\
\text { reduced milk intake }\end{array}$ & $\begin{array}{l}\text { Normal white blood cell parameters, } \\
\text { LFT, CK, immunoglobulin }\end{array}$ & Opacities & Positive anal, pharyngeal samples & Supportive & Alive \\
\hline Buonsenso & None (2) & ND (2) & ND (2) & Positive nasopharyngeal samples & $\begin{array}{l}\text { Supportive } \\
\text { (1) }\end{array}$ & All alive \\
\hline Pu Yang. & None (6), vomiting (1) & Elevated cytokines (5) & Evidence of RDS (5) & $\begin{array}{l}\text { Negative throat, amniotic fluid, cord } \\
\text { blood samples }\end{array}$ & $\begin{array}{l}\text { Supportive } \\
\text { (7) }\end{array}$ & All alive \\
\hline Alzamora. & Dyspnea, cough & $\begin{array}{l}\text { Normal white blood cell parameters, } \\
\text { immunoglobulin }\end{array}$ & Normal & Positive nasopharyngeal sample & $\begin{array}{l}\text { Supportive, } \\
\text { oxygen }\end{array}$ & \\
\hline Noelle & None & ND & ND & Negative nasopharyngeal & $\begin{array}{l}\text { Supportive, } \\
\text { oxygen }\end{array}$ & Alive \\
\hline Jie Yan & None & ND & ND & Negative & Oxygen (1) & $\begin{array}{l}\text { Alive } \\
(115) \\
\text { Fetal } \\
\text { death (1) }\end{array}$ \\
\hline Yan Ting wu & None (29) Fever (1) & $\begin{array}{l}\text { Leukocytosis (5) Neutrophil (5) ND } \\
\text { (25) }\end{array}$ & Opacities (5) & Positive nasopharyngeal samples (2) & $\begin{array}{l}\text { Supportive } \\
\text { (5) } \\
\text { Interferon } \\
\text { (3) None } \\
\text { (25) }\end{array}$ & All alive \\
\hline $\mathrm{Na} \mathrm{Li}$ & ND & ND & ND & Negative throat samples & Supportive & All alive \\
\hline Dehan Liu & ND & ND & ND & Negative nasopharyngeal samples & None & All alive \\
\hline Eun-Kyung Lee & None & Normal white cell parameters & Normal & $\begin{array}{l}\text { Negative nasopharyngeal, } \\
\text { oropharyngeal, stool, urine, amiotic } \\
\text { fluid, placenta, peripheral blood and } \\
\text { cord blood samples }\end{array}$ & None & Alive \\
\hline $\begin{array}{l}\text { Fatemeh } \\
\text { Eghbalian }\end{array}$ & None & Normal white cell parameters, LFT & Normal & $\begin{array}{l}\text { Positive oropharyngeal, stool, saliva, } \\
\text { plasma, urine, anal, throat, rectal, } \\
\text { pharyngeal, amniotic fluid, breast milk, }\end{array}$ & None & Alive \\
\hline
\end{tabular}




\begin{tabular}{|c|c|c|c|c|c|c|}
\hline First Author & Clinical features & Laboratory findings & Radiological findings & Diagnosis of COVID-19 & Treatment & Outcome \\
\hline & & & & \multicolumn{3}{|l|}{ peripheral blood, cord blood } \\
\hline $\begin{array}{l}\text { Gregorio- } \\
\text { Hernández }\end{array}$ & None & ND & $\begin{array}{l}\text { thin pleural line with } \\
\text { conserved lung sliding (1), B- } \\
\text { lines, consolidation (1), A } \\
\text { pattern, B lines, superficial } \\
\text { consolidation (1) }\end{array}$ & $\begin{array}{l}\text { Positive nasopharyngeal samples (2) } \\
\text { Positive bronchoalveolar aspirate (1) }\end{array}$ & $\begin{array}{l}\text { None (2) } \\
\text { Supportive } \\
\text { (1) Oxygen } \\
\text { (1) }\end{array}$ & All alive \\
\hline $\begin{array}{l}\text { Serafina } \\
\text { Perrone }\end{array}$ & None & ND & ND & $\begin{array}{l}\text { Negative nasopharyngeal and breast } \\
\text { milk samples }\end{array}$ & $\begin{array}{l}\text { Supportive } \\
\text { (1) Oxygen } \\
\text { (1) }\end{array}$ & Alive \\
\hline Mahdavi & $\begin{array}{l}\text { Fever, dyspnea, lethargy, } \\
\text { cyanosis, cough, reduced milk } \\
\text { intake, nasal congestion }\end{array}$ & Normal white cell parameters & $\begin{array}{l}\text { Consolidation with air } \\
\text { bronchograms }\end{array}$ & Positive nasopharyngeal sampl & $\begin{array}{l}\text { Supportive, } \\
\text { oxygen, } \\
\text { antibiotic, } \\
\text { antiviral }\end{array}$ & Alive \\
\hline Rishi Lumba. & None & $\begin{array}{l}\text { Neutrophilia, normal cytokine, LFT, CK, } \\
\text { immunoglobulin }\end{array}$ & Normal & $\begin{array}{l}\text { Negative nasopharyngeal, } \\
\text { oropharyngeal, stool, saliva, plasma, } \\
\text { urine, anal, throat, rectal, pharyngeal, } \\
\text { amniotic fluid, breast milk, peripheral } \\
\text { blood, cord blood }\end{array}$ & Antibiotic & Alive \\
\hline Salik & $\begin{array}{l}\text { Fast breathing, cyanosis, } \\
\text { lethargy, feed intolerance }\end{array}$ & ND & $\begin{array}{l}\text { Bilateral pulmonary granular } \\
\text { opacities and reduced lung } \\
\text { volumes }\end{array}$ & Positive nasopharyngeal sample & None & Alive \\
\hline $\begin{array}{l}\text { Soumeth } \\
\text { Abasse }\end{array}$ & None & $\begin{array}{l}\text { Normal white cell parameters and LFT, } \\
\text { increased CK }\end{array}$ & $\begin{array}{l}\text { Ground glass opacities and } \\
\text { consolidation }\end{array}$ & Positive nasopharyngeal sample & None & Alive \\
\hline Ying Xion. & Not available & $\begin{array}{l}\text { Neutrophilia, lymphopenia, increased } \\
\text { cytokines }\end{array}$ & $\begin{array}{l}\text { Bronchovascular shadows } \\
\text { and ground-glass opacity }\end{array}$ & Positive oropharyngeal sample & None & Alive \\
\hline
\end{tabular}

ND: Not documented; LFT: Liver function test; Creatine kinase; 


\section{CLINICAL AND LABORATORY FEATURES}

The following are clinical and/or laboratory examinations and results that were described by the studies.

\section{CLINICAL FEATURES}

1. Systemic features: Thirteen studies reported neonatal fever after birth to a COVID-19 positive mother with a total of 17 neonates. ${ }^{1,2,17,21,22,30,35,36,38,44,46,48,54}$ Four studies showed that four neonates had lethargy. $1,17,20,44$ Swollen superficial lymph nodes was identified in a COVID-19 positive male neonate, ${ }^{7}$ and hypothermia was reported in one neonate (Table 3 ). ${ }^{3}$

2. Respiratory features reported include cough (four neonates), ${ }^{2,4,17,36}$ nasal discharge, congestion and stuffiness (6 neonates), ${ }^{1-3,17,30}$ tachypnoea (21 neonates), $1,3,5,7,28,31,38,44-47$ respiratory distress syndrome (5 neonates), ${ }^{42,44}$ mild respiratory distress (3 neonates), 1,4,32 moaning (3 neonates), 5,45 asphyxia (4 neonates), ${ }^{38,44,59}$ and sneezing (1 neonate) (Table 3) ${ }^{7}$

3. Cardiovascular features include tachycardia in 5 neonates, cyanosis in 12 neonates, dyspnoea in 9 neonates $1,4,17,28,31,36,38,45$ and hypotension in one neonate (Table 3 ). ${ }^{3}$

4. Skin/dermatological features reported include cutaneous mottling (one neonate), ${ }^{1}$ whole body jaundice (one neonate), ${ }^{2}$ rash (3 neonates), ${ }^{45,46}$ ruddy skin colour (2 neonates) $)^{7,8}$ and skin ulceration (one neonate) (Table 3$).{ }^{45}$

5. Gastrointestinal features: Abdominal distension was reported in 3 neonates, ${ }^{28,46,48}$ reduced feeding and feeding intolerance in 8 neonates, $3,7,22,28,44,46$ and reduced milk intake in 8 neonates. $3,7,17,21,28,46,50,54$ Milk refusal, bloating and gastric bleeding was reported among COVID-19 negative neonates born to positive mothers in one study. ${ }^{46}$ Nine studies reported vomiting in nine neonate $2,7,8,30,36,42,44,46,54$ (Table 3) while diarrhoea was reported in two neonates in two studies. $^{7,54}$

\section{RADIOLOGICAL AND LABORATORY RESULTS}

1. SARS-COV-2 testing following childbirth: 38 (9.0\%) neonates had PCR confirmed COVID-19 infection. For majority of the babies, nasopharyngeal swab was used for testing.

2. Radiological features reported include bilateral perihilar streaking (one neonate), ${ }^{21}$ ground glass opacities (12 neonates, $, 3,7,20,27,31,32,35,60$ consolidation (3 neonates), $3,17,32$ patchy fuzzy shadows (one neonate), ${ }^{54}$ bilateral streaky infiltrates (one neonate), ${ }^{34}$ pneumothorax (one neonate), ${ }^{46}$ reduced lung volume(two neonates), 5,20 thickened lung texture (one neonate),${ }^{8}$ diffuse haziness (one neonate), ${ }^{48}$ mild pulmonary infection (two neonates), ${ }^{46,47}$ pneumonia (4 neonates), ${ }^{44,48}$ Respiratory distress syndrome (RDS) (4 neonates), 38,42,46 Bronchovascular shadows ( 2 neonates), ${ }^{46,60}$ increased lung markings

\section{(3 neonates) (Table 4). ${ }^{36}$}

3. Other laboratory findings identified include: Elevated white blood cell (WBC) count (10 noenates), 35,44,45,58 Abnormal Liver Function Test (LFT) (four neonates), 8,21,46 Elevated cytokines (two neonates), $3,42,58$ elevated creatine kinase (nine), $8,30,39,42,44$ Lymphopenia (6 neonates) (Table 4). $8,14,34,48,55$

\section{TRANSMISSION}

Among the 38 neonates that tested positive, vertical transmission was reported in 11 neonates, while horizontal transmission was reported in 17 babies. The route of transmission was not clear in the remaining babies (Table 5).

\section{TREATMENT RECEIVED}

Treatment was supportive for majority of the babies. Few received antiviral (9 neonates), 1,9,17,21,47 antibiotics 23 neonates), 1,3,5,8,9,17,19,21,22,27,28,30,31,35,37,38,44,48 and oxygen therapy/ventilation (22 neonates) (Table 4). $4,5,17,21,23,26-29,31,34,38,42,44,45,59$

\section{OUTCOMES AND COMPLICATIONS}

The complications reported include pneumonia, pneumothorax, refractory shock, and hypoxic respiratory failure. Neonates who tested positive were discharged after they tested negative to the virus. All the COVID-19 positive babies survived (Table 2). Three of the studies reported neonatal death in 5 neonates but these babies were not tested before death. ${ }^{14,46,59}$

\section{DISCUSSION}

The review examined studies that looked at the exposure to, transmission and infection of neonates as well as clinical manifestation, laboratory findings and outcomes of neonates whose mothers had COVID-19 infection. Few neonates of mothers with COVID-19 infection in this review tested positive to the virus. This finding does not support vertical transmission as a major mode of infection of the newborn. It should be noted however that quite a number of the neonates were delivered through caesarean section and the health care workers may have observed adequate infection prevention and control measures. Some of the neonates who had positive results had close contacts with other family members diagnosed with coronavirus and possibly got the infection from person-to-person transmission. ${ }^{1-3}$ Wang et $\mathrm{al}^{8}$ reported a positive test of COVID-19 in a 36 hour-old neonate in Wuhan, China. A possibility of nosocomial infection was entertained by the authors because the initial cord blood and placenta test results were negative for severe acute respiratory syndrome corona virus 2 (SARS-CoV-2). However, the finding of positive nasopharyngeal and anal swabs among 3 out of 33 neonates whose mothers had COVID-19 pneumonia by day 2 of life by Zeng et $\mathrm{al}^{44}$ raises the possibility of vertical transmission. Duran et $\mathrm{al}^{61}$ in a systematic review of neonatal cases also did not 
Table 3: Summary of neonatal clinical presentations by number of studies

Clinical Features

\begin{tabular}{|c|c|c|c|}
\hline & & $\begin{array}{l}\text { Number } \\
\text { of studies }\end{array}$ & $\begin{array}{l}\text { Number of babies (Proportion of exposed and } \\
\text { positive babies with symptoms) }\end{array}$ \\
\hline \multirow[t]{5}{*}{$\begin{array}{l}\text { Systemic } \\
\text { Features }\end{array}$} & Fever $1,2,17,21,22,30,35,36,38,44,46,48,54$ & 13 & $17(4.1 \%)$ \\
\hline & Lethargy $1,17,20,44$ & 4 & $4(1.0 \%)$ \\
\hline & Lymphadenopathy $^{7}$ & 1 & $1(0.2 \%)$ \\
\hline & Hypothermia ${ }^{3}$ & 1 & $1(0.2 \%)$ \\
\hline & Edema $^{45}$ & 1 & $2(0.5 \%)$ \\
\hline \multicolumn{4}{|c|}{$\begin{array}{l}\text { Respiratory } \\
\text { Features }\end{array}$} \\
\hline & Cough $2,4,17,36$ & 4 & $4(1.0 \%)$ \\
\hline & $\begin{array}{l}\text { nasal discharge, nasal congestion and } \\
\text { stuffiness }{ }^{1-3,17,30}\end{array}$ & 6 & $6(1.4 \%)$ \\
\hline & Tachpynoea $1,3,5,7,20,28,31,38,44-47$ & 12 & $21(5.1 \%)$ \\
\hline & $\mathrm{RDS}^{42,44}$ & 2 & $5(1.2 \%)$ \\
\hline & Mild respiratory distress ${ }^{1,4,32}$ & 3 & $3(0.7 \%)$ \\
\hline & Moaning/Mild grunting 5,42 & 2 & $3(0.7 \%)$ \\
\hline & Asphyxia $38,44,59$ & 3 & $4(1.0 \%)$ \\
\hline & Sneezing 7 & 1 & $1(0.2 \%)$ \\
\hline \multicolumn{4}{|c|}{$\begin{array}{l}\text { Cardiovascular } \\
\text { Features }\end{array}$} \\
\hline & Tachycardia $1-3,22,46$ & 5 & $5(1.2 \%)$ \\
\hline & Dyspnoea $^{1,4,17,28,31,36,38,45}$ & 8 & $9(2.2 \%)$ \\
\hline & Cyanosis $3,17,20,21,27,28,31,38,44,46$ & 10 & $12(2.9 \%)$ \\
\hline & Hypotension ${ }^{3}$ & 1 & $1(0.2 \%)$ \\
\hline \multicolumn{4}{|c|}{$\begin{array}{l}\text { Skin/ } \\
\text { Dermatological } \\
\text { features }\end{array}$} \\
\hline & Cutaneous mottling ${ }^{1}$ & 1 & $1(0.2 \%)$ \\
\hline & Whole body Jaundice 2 & 1 & $1(0.2 \%)$ \\
\hline & $\operatorname{Rash}^{45,46}$ & 2 & $3(0.7 \%)$ \\
\hline & Ruddy Skin colour 7,8 & 2 & $2(0.5 \%)$ \\
\hline & Skin Ulceration 45 & 1 & $1(0.2 \%)$ \\
\hline \multicolumn{4}{|c|}{$\begin{array}{l}\text { Gastrointestinal } \\
\text { Features }\end{array}$} \\
\hline & Abdominal Distension28,46,48 & 3 & $3(0.7 \%)$ \\
\hline & $\begin{array}{l}\text { Reduced feeding and feeding } \\
\text { intolerance } 3,7,22,28,44,46\end{array}$ & 6 & $8(1.9 \%)$ \\
\hline & $\begin{array}{l}\text { Reduced milk } \\
\text { intake } 3,7,17,21,28,46,50,54\end{array}$ & 8 & $8(1.9 \%)$ \\
\hline & Bloating and gastric bleeding 46 & 1 & $1(0.2 \%)$ \\
\hline & Vomiting $2,7,8,30,36,42,44,46,54$ & 9 & $9(2.2 \%)$ \\
\hline & Diarrhoea 7,54 & 2 & $2(0.5 \%)$ \\
\hline
\end{tabular}

find any evidence of vertical transmission of corona virus.

Most of the neonates who were positive for SARS-CoV-2 had mild clinical symptoms. Mild symptoms according to World Health Organisation (WHO) interim guideline is when the patient has uncomplicated upper respiratory tract viral infection with non-specific symptoms such as fever, fatigue, cough, malaise and rarely may present with diarrhea, nausea, and vomiting. ${ }^{62}$ This finding is similar to reports in older children who also presented with mild or moderate clinical symptoms. ${ }^{63,64}$ The findings of cough, tachypnea, nasal discharge in neonates who tested positive for COVID-19 observed in this review is in keeping with findings in some studies carried out among older children and adults. ${ }^{65-67}$ 
Table 4: Laboratory features, Radiological features, and Treatment

\begin{tabular}{|c|c|c|}
\hline Features & $\begin{array}{l}\text { Number of } \\
\text { Studies }\end{array}$ & $\begin{array}{l}\text { Number of Neonates (Proportion of exposed and } \\
\text { positive babies with symptoms) }\end{array}$ \\
\hline \multicolumn{3}{|l|}{ Radiological Features } \\
\hline Bilateral perihilar streaking 21 & 1 & $1(0.2 \%)$ \\
\hline Ground glass opacities $3,7,20,27,31,32,35,60$ & 8 & $12(2.9 \%)$ \\
\hline Consolidation $3,17,32$ & 3 & $3(0.7 \%)$ \\
\hline Patchy fuzzy shadows ${ }^{54}$ & 1 & $1(0.2 \%)$ \\
\hline Bilateral streaky infiltrates 34 & 1 & $1(0.2 \%)$ \\
\hline Pneumothorax 46 & 1 & $1(0.2 \%)$ \\
\hline Reduced lung volume 5,20 & 2 & $2(0.5 \%)$ \\
\hline Thickened lung texture 8 & 1 & $1(0.2 \%)$ \\
\hline Diffuse haziness 48 & 1 & $1(0.2 \%)$ \\
\hline Mild pulmonary infection 46,47 & 2 & $2(0.5 \%)$ \\
\hline Pneumonia 44,48 & 2 & $4(1.0 \%)$ \\
\hline $\mathrm{RDS}^{38,42,46}$ & 3 & $4(1.0 \%)$ \\
\hline Bronchovascular shadows 46,60 & 2 & $2(0.5 \%)$ \\
\hline Increased Lung markings ${ }^{36}$ & 1 & $3(0.7 \%)$ \\
\hline \multicolumn{3}{|l|}{ Laboratory Features } \\
\hline Elevated WBC count $35,44,45,58$ & 4 & $10(2.4 \%)$ \\
\hline Abnormal LFT $8,21,46$ & 3 & $4(1.0 \%)$ \\
\hline Elevated Cytokines $3,42,58$ & 3 & $3(0.7 \%)$ \\
\hline Elevated Creatine Kinase $8,30,39,42,44$ & 5 & $9(2.2 \%)$ \\
\hline Lymphopenia $8,14,34,48,55$ & 5 & $6(1.4 \%)$ \\
\hline \multicolumn{3}{|l|}{ Treatment } \\
\hline Antiviral $1,9,17,21,47$ & 5 & $9(2.2 \%)$ \\
\hline Interferon 35,54 & 2 & $4(1.0 \%)$ \\
\hline Antibiotics $1,3,5,8,9,17,19,21,22,27,28,30,31,35,37,38,44,48$ & 18 & $23(5.5 \%)$ \\
\hline $\begin{array}{l}\text { Oxygen/ } \\
\text { ventilation } 3-5,17,21,23,26-29,31,34,38,42,44,45,59\end{array}$ & 17 & $22(5.3 \%)$ \\
\hline
\end{tabular}

WBC: White blood cell; RDS: Respiratory distress syndrome

Table 5: Modes of neonatal COVID-19 exposures and transmissions in included studies

\begin{tabular}{lll}
\hline Mode of transmission & Number of Studies & Number of babies \\
\hline Horizontal (Person to Person) ${ }^{1-3,7,14,21,22,25,27,28,31,34,36,38,39,54}$ & 16 & 17 \\
\hline Vertical transmission $4,35-37,59$ & 5 & 11 \\
Uncertain about mode of transmission $8,15,29,44,47$ & 3 & 10 \\
\hline
\end{tabular}

Lymphopenia observed among these neonates has also been reported in older paediatric age groups. ${ }^{68}$ In adults with COVID -19 infection, lymphopenia is associated with poor prognosis and is an indicator of severe disease. ${ }^{68-70}$ Possible explanations for lymphopenia include direct infection of the lymphocytes, damage to lymphoid organs, disruption of activities of cytokines resulting in apoptosis of lymphocytes. ${ }^{70}$ Fever was a significant finding in newborns delivered to COVID-19 positive mothers, despite the fact that quite a number of these babies were negative. This could be as a result of maternal cytokines transferred passively to the fetus and evoking immune response in the neonatal period. The implication of this finding is the fact that the presence of fever in a newborn during this period of COVID-19 pandemic may be a good reason to screen mothers for SARS-CoV-2 infection. In adult populations, fever was also a frequent finding alongside fatigue and cough during presentation in the hospital. 65,66

Gastrointestinal symptoms appear to be a common finding in neonates who were positive for COVID-19. These 
have also been reported among other paediatric age groups. ${ }^{71}$ Symptoms such as reduced feeding, vomiting and diarrhea may be due to the body's response to acute viral replication in the gastrointestinal tract. Therefore, the presence of gastrointestinal features in a neonate born to a COVID-19 positive mother or who may have been exposed to a COVID -9 positive individual, may raise a suspicion of COVID-19 infection in the neonate. SARS-COV-2 RNA has been demonstrated in stool samples of hospitalized patients with COVID-19 with some still having positive stool test even after respiratory samples have tested negative, thereby posing a risk of feco-oral transmission. ${ }^{72}$

There is currently no definitive drug treatment for COVID-19 infection. In this review, supportive therapy which includes administration of intravenous fluids, intranasal oxygen were the mainstay of treatment for COVID-19 positive neonates because many of them had mild clinical symptoms. Some of the neonates received intravenous antibiotics as a way of preventing secondary bacterial infection. A few studies documented the use of antiviral drugs like oseltamivir and acyclovir. $1,9,14,18$ The effect of these drugs in the clinical course of COVID-19 infection is still being evaluated. In this review most of the neonates did not receive antiviral drugs and still had good outcome. The use of oseltamivir has been reported among adults with COVID-19 infection without significant outcome or change in disease progression. ${ }^{65}$ Chloroquine and hydroxychloroquine have been demonstrated to have in vitro activity against SARS-CoV-2. ${ }^{73}$ The combination of hydroxychloroquine and azithromycin has been reported to improve clinical outcomes among adult population in France, ${ }^{74}$ though this was a retrospective study and therefore has its limitations. In a randomized control trial in Spain among adult population with mild COVID-19 infection, no benefit was observed with use of hydroxychloroquine. ${ }^{75}$ Recently the U.S Food and Drug Administration (FDA) issued emergency use authorization of remdesivir for the treatment of suspected or laboratory confirmed severe cases of COVID-19 in adult and children. ${ }^{76}$ Therefore, more clinical trials are needed to determine the effective drug treatment for COVID-19 in both adults and children.

Most of the neonates born to COVID- 19 mothers had good outcomes. This finding gives credence to the fact that the infection is mild in neonates. Since there is currently no evidence of transmission of the virus in breast milk and because of the mild illness in this age group, WHO interim guideline recommends that infants born to mothers with suspected, probable or confirmed COVID-19 should be fed according to standard infant feeding guidelines, while ap- plying necessary precautions for infection prevention and control. ${ }^{62}$

\section{STRENGTHS AND LIMITATIONS}

One of the strengths of this study is that it's a comprehensive global review. Secondly, it included both babies that tested negative though exposed to COVID-19 positive mothers and babies that tested positive. One limitation of this review is that it included mainly case reports and case series, which do not provide high quality evidence; however, these are the major kinds of research available considering the fact that neonatal COVID-19 is a new and evolving disease.

\section{CONCLUSIONS}

The clinical manifestations of COVID-19 in neonates are mild and outcomes are better than in adult population. There is currently little evidence of vertical transmission in neonates. Given immunological susceptibility of neonates and the fact that COVID-19 is a newly emerged and evolving disease, it is important to continue in-depth research using higher quality study designs and methodologies.

\section{FUNDING}

This research did not receive any specific grant from funding agencies in the public, commercial, or not-for-profit sectors.

\section{AUTHORSHIP CONTRIBUTIONS}

OBE, CDO: study conceptualization and design. OBE, ICA, INO, CDO, OUA, OWD, EE: data extraction, analysis and interpretation of results, manuscript drafting and approval of the final manuscript for publication.

\section{COMPETING INTERESTS}

The authors completed the Unified Competing Interest form at www.icmje.org/coi_disclosure.pdf (available upon request from the corresponding author), and declare no conflicts of interest.

Submitted: September 11, 2020 GMT, Accepted: September 14, 2020 GMT 


\section{REFERENCES}

1. Kamali Aghdam M, Jafari N, Eftekhari K. Novel coronavirus in a 15-day-old neonate with clinical signs of sepsis, a case report. Infect Dis. 2020;52(6):427-429. doi:10.1080/23744235.2020.1747 $\underline{634}$

2. Han MS, Seong M-W, Heo EY, et al. Sequential analysis of viral load in a neonate and her mother infected with SARS-CoV-2. Infect Dis Soc Am. 2020:1-13. doi:10.1093/cid/ciaa447/5820869

3. Coronado Munoz A, Nawaratne U, McMann D, Ellsworth M, Meliones J, Boukas K. Late-Onset Neonatal Sepsis in a Patient with Covid-19. N Engl J Med. 2020;382(19):e49. doi:10.1056/neimc2010614

4. Alzamora MC, Paredes T, Caceres D, Webb CM, Valdez LM, La Rosa M. Severe COVID-19 during Pregnancy and Possible Vertical Transmission. Am J Perinatol. 2020;37(08):861-865. doi:10.1055/s-0040-1 $\underline{710050}$

5. Peng Z, Wang J, Mo Y, et al. Unlikely SARS-CoV-2 vertical transmission from mother to child: A case report. J Infect Public Health. 2020;13(5):818-820. do i:10.1016/j.jiph.2020.04.004

6. Xiong X, Wei H, Zhang Z, et al. Vaginal Delivery Report of a Healthy Neonate Born to a Convalescent Mother with COVID19. J Med Virol. 2020;92(9):1657-1659. doi:10.1002/jmv.25857

7. Zeng L, Tao X, Yuan W, Wang J, Liu X, Liu Z. The First Newborn Coronavirus Pneumonia in China. Chinese J Pediatr. 2020;58(04):279-280.

8. Wang S, Guo L, Chen L, et al. A Case Report of Neonatal 2019 Coronavirus Disease in China. Clin Infect Dis. 2020;71(15):853-857. doi:10.1093/cid/ciaa2 $\underline{25}$

9. Chen S, Liao E, Cao D, Gao Y, Sun G, Shao Y. Clinical analysis of pregnant women with 2019 novel coronavirus pneumonia. J Med Virol. 2020;92(9):1556-1561. doi:10.1002/imv.25789

10. Shamseer L, Moher D, Clarke M, et al. Preferred reporting items for systematic review and metaanalysis protocols (PRISMA-P) 2015: Elaboration and explanation. BMJ. 2015;349(jan02 1):g7647-g7647. do i:10.1136/bmj.g7647
11. Wells G, Shea B, O’Connell D, Peterson J, Welch V, Losos M. The Newcastle-Ottawa Scale (NOS) for assessing the quality of nonrandomised studies in meta-analyses. 2019. http://www.ohri.ca/programs/cl inical_epidemiology/oxford.asp. Accessed August 12, 2020.

12. National Heart Lung and Blood Institute. Quality Assessment Tool for Observational Cohort and CrossSectional Studies. 2017. https://www.nhlbi.nih.gov/he alth-topics/study-quality-assessment-tools. Accessed August 30, 2020.

13. Murad MH, Sultan S, Haffar S, Bazerbachi F. Methodological quality and synthesis of case series and case reports. https://pubmed.ncbi.nlm.nih.gov/29 420178/. Accessed August 25, 2020.

14. Hantoushzadeh S, Shamshirsaz AA, Aleyasin A, et al. Maternal death due to COVID-19. Am J Obs Gynecol. 2020;223(1):109.e1-109.e16. doi:10.1016/i.aj og.2020.04.030

15. Zamaniyan M, Ebadi A, Aghajanpoor S, Rahmani Z, Haghshenas M, Azizi S. Preterm delivery in pregnant woman with critical COVID-19 pneumonia and vertical transmission. Prenat Diagn. June 2020. $\underline{\mathrm{d}}$ oi:10.1002/pd.5713

16. Eghbalian F, Esfahani AM, Jenabi E. COVID-19 Virus in a 6-Day-Old Girl Neonate: A Case Report. Clin Pediatr (Phila. 2020;00(0):1-2.

17. Mahdavi S, Kheirieh A, Daliri S, et al. More reliability of suspicious symptoms plus chest CT-scan than RT_PCR test for the diagnosis of COVID-19 in an 18-days-old neonate. IDCases. 2020;21:e00905. doi:1 0.1016/i.idcr.2020.e00905

18. Lee E-K, Kim WD, Lee D won, Lee S-A. Management of the first newborn delivered by a mother with COVID-19 in South Korea. Clin Exp Pediatr. 2020;63(9):373-375. doi:10.3345/cep.2020.00 $\underline{850}$

19. Lumba R, Remon J, Louie M, et al. Neonate Born to a Mother with a Diagnosis of Suspected IntraAmniotic Infection versus COVID-19 or Both. Case Rep Pediatr. 2020;2020:19-21. doi:10.1155/2020/8886 $\underline{800}$

20. Salik I, Mehta B. Tetralogy of Fallot palliation in a COVID-19 positive neonate. J Clin Anaesth. 2020;66:109914. doi:10.1016/i.jclinane.2020.109914 
21. Patek P, Corcoran J, Adams L, Khandhar P. SARSCoV-2 Infection in a 2-Week-Old Male with Neutropenia. Clin Pediatr (Phila). 2020;59(9-10):918-920. doi:10.1177/00099228209200 14

22. Dumpa V, Kamity R, Vinci AN, Noyola E, Noor A. Neonatal Coronavirus 2019 (COVID-19) Infection: A Case Report and Review of Literature. Cureus. 2020;12(5):e8165. doi:10.7759/cureus.8165

23. Breslin N, Baptiste C, Gyamfi-Bannerman C, et al. Coronavirus disease 2019 infection among asymptomatic and symptomatic pregnant women: Two weeks of confirmed presentations to an affiliated pair of New York City hospitals. Am J Obs Gynecol MFM. 2020;2(2):100118. doi:10.1016/j.ajogmf.2020.10 $\underline{0118}$

24. Iqbal SN, Overcash R, Mokhtari N, et al. An Uncomplicated Delivery in a Patient with Covid-19 in the United States. N Engl J Med. 2020;382(16):e34. do $\mathrm{i}: 10.1056 /$ neimc2007605

25. Costa S, Buonsenso D, Sanguinetti M, et al. Neonatal Late Onset Infection with Severe Acute Respiratory Syndrome Coronavirus 2. Am J Perinatol. 2020;37(08):869-872. doi:10.1055/s-0040-1710541

26. Perrone S, Giordano M, Meoli A, et al. Lack of viral transmission to preterm newborn from a COVID - 19 positive breastfeeding mother at 11 days postpartum. J Med Virol. 2020;1-2. doi:10.1002/jmv.26037

27. Sinelli M, Paterlini G, Citterio M, Di Marco A, Fedeli T, Ventura ML. Early neonatal SARS-CoV-2 infection manifesting with hypoxemia requring respiratory support. Pediatrics. 2020;146(1):e20201121. doi:10.1542/peds.2020-1121

28. Ferrazzi E, Frigerio L, Savasi V, et al. Vaginal delivery in SARS - CoV - 2 - infected pregnant women in Northern Italy: A retrospective analysis. BJOG. 2020;127(9):1116-1121. doi:10.1111/1471-052 $\underline{8.16278}$

29. Gregorio-Hernández R, Escobar-Izquierdo AB, Cobas-Pazos J, Martínez-Gimeno A. Point-of-care lung ultrasound in three neonates with COVID-19. Eur J Pediatr. 2020;179(8):1279-1285. doi:10.1007/s00 431-020-03706-4

30. Chacón-Aguilar R, Osorio-Cámara JM, SanjurjoJimenez I, González-González C, López-Carnero J, Pérez-Moneo B. COVID-19: Fever syndrome and neurological symptoms in a neonate. An Pediatr (Eng Ed). 2020;92(6):373-374.
31. Díaz CA, Maestro ML, Pumarega MTM, Antón BF, Alonso CP. First case of neonatal infection due to COVID-19 in Spain. An Pediatr (Engl Ed). 2020;92(4):237-238.

32. Abasse S, Essabar L, Costin T, et al. Neonatal COVID-19 Pneumonia: Report of the First Case in a Preterm Neonate in Mayotte, an Overseas Department of France. Children. 2020;7(8):87. doi:1 $\underline{0.3390 / \text { children7080087 }}$

33. Lowe B, Bopp B. COVID - 19 vaginal delivery - A case report. Aust N Z J Obstet Gynaecol. 2020;60(3):465-466. doi:10.1111/ajo.13173

34. Piersigilli F, Carkeek K, Hocq C, et al. Case Report COVID-19 in a 26-week preterm neonate. Lancet Child Adolesc Heal. 2020;4(6):476-478. doi:10.1016/s 2352-4642(20)30140-1

35. Wu Y-T, Liu J, Xu J-J, et al. Neonatal outcome in 29 pregnant women with COVID-19: A retrospective study in Wuhan, China. PLoS Med. 2020;17(7):e1003195. doi:10.1371/journal.pmed.1003 195

36. Zhang Z-J, Yu X-J, Fu T, et al. Novel coronavirus infection in newborn babies aged $<28$ days in China. Eur Respir J. 2020;55(6):2000697. doi:10.1183/139930 $\underline{03.00697-2020}$

37. Hu X, Gao J, Luo X, et al. Severe Acute Respiratory Syndrome Transmission in Neonates Born to Mothers With Coronavirus Disease 2019. Obstet Gynecol. 2020;136(1):1-3.

38. Sun M, Xu G, Yang Y, et al. Evidence of mother-tonewborn infection with COVID-19. Br J Anaesth. 2020;125(2):e245-e247. doi:10.1016/j.bja.2020.04.066

39. Chen H, Guo J, Wang C, et al. Clinical characteristics and intrauterine vertical transmission potential of COVID-19 infection in nine pregnant women: A retrospective review of medical records. Lancet. 2020;395(10226):809-815. doi:10.1016/s014 $\underline{0-6736(20) 30360-3}$

40. Liu D, Li L, Wu X, et al. Pregnancy and Perinatal Outcomes of Women With Coronavirus Disease (COVID-19) Pneumonia: A Preliminary Analysis. AJR. 2020;215(1):127-132. doi:10.2214/ajr.20.23072

41. Li N, Han L, Peng M, et al. Maternal and neonatal outcomes of pregnant women with COVID-19 pneumonia: A case-control study. https://academic.o up.com/cid/article-abstract/doi/10.1093/cid/ciaa352/5 813589. Accessed April 15, 2020. 
42. Yang P, Wang X, Liu P, et al. Clinical characteristics and risk assessment of newborns born to mothers with COVID-19. J Clin Virol. 2020;127:104356. doi:10.1016/j.jcv.2020.104356

43. Liu W, Wang J, Li W,et al. Clinical characteristics of 19 neonates born to mothers with. Front Med. 2020;14(2):193-198.

44. Zeng L, Xia S, Yuan W, et al. Neonatal Early-Onset Infection With SARS-CoV-2 in 33 Neonates Born to Mothers With COVID-19 in Wuhan, China. JAMA Pediatr. 2020;23(77):4-6. doi:10.1001/jamapediatric $\underline{\mathrm{s} .2020 .0878}$

45. Chen Y, Peng H, Wang L, et al. Infants Born to Mothers With a New Coronavirus (COVID-19). Front Pediatr. 2020;8(104):1-5. doi:10.3389/fped.2020.0010 $\underline{4}$

46. Zhu H, Wang L, Fang C, et al. Clinical analysis of 10 neonates born to mothers with 2019-nCoV pneumonia. Transl Pediatr. 2020;9(1):51-60. doi:10.2 1037/tp.2020.02.06

47. Yu N, Li W, Kang Q, et al. Clinical features and obstetric and neonatal outcomes of pregnant patients with COVID-19 in Wuhan, China: A retrospective, single-centre, descriptive study. Lancet Infect Dis. 2020;20(5):559-564. doi:10.1016/s1473-3099(20)3017 $\underline{6-6}$

48. Fan C, Lei D, Fang C, et al. Perinatal Transmission of COVID-19 Associated SARS-CoV-2: Should We Worry? Infect Dis Soc Am Am. March 2020. doi:10.10 93/cid/ciaa226

49. Li Y, Jing W, Jingjing Z. A case of asymptomatic new coronavirus infection in late pregnancy. Chinese J Perinat Med. 2020;23. doi:10.3760/cma.j.cn113903-2 $\underline{0200221-00143}$

50. Siying Z, Juanjuan G, Yuming C, et al. A case of new coronavirus infection in perinatal period. Chinese J Perinat Med. 2020;23(2):85-90.

51. Bailiang B, Zhongliang G, Shuangying H, et al. A case of multi-site pathogenic detection of new coronavirus pneumonia mothers and their newborns. Chinese J Neonatol. 2020;35(2):85-86.

52. Khan S, Jun L, Siddique R, et al. Association of COVID-19 with pregnancy outcomes in health-care workers and general women. Clin Microbiol Infect. 2020;26(6):788-790. doi:10.1016/i.cmi.2020.03.034

53. Lu D, Sang L, Du S, Li T, Chang Y, Yang X. Asymptomatic COVID - 19 infection in late pregnancy indicated no vertical transmission. J Med Virol. 2020;92(9):1660-1664. doi:10.1002/imv.25927
54. Wang J, Wang D, Chen G-C, Tao X-W, Zeng L-K. SARS-CoV-2 infection with gastrointestinal symptoms as the first manifestation in a neonate. Zhongguo Dang Dai Er Ke Za Zhi. 2020;22(3):211-214.

55. Wu Y, Liu C, Dong L, et al. Coronavirus disease 2019 among pregnant Chinese women: Case series data on the safety of vaginal birth and breastfeeding. BJOG. 2020;127(9):1109-1115. doi:10.1111/1471-052 $\underline{8.16276}$

56. Wang X, Zhou Z, Zhang J, Zhu F, Tang Y, Shen X. A case of 2019 Novel Coronavirus in a pregnant woman with preterm delivery. Clinical Infectious Diseases. 2020;71(15):844-846. doi:10.1093/cid/ciaa2 $\underline{00}$

57. Zeng $\mathrm{H}, \mathrm{Xu} \mathrm{C}$, Fan J, et al. Antibodies in Infants Born to Mothers With COVID-19 Pneumonia. JAMA. March 2020. doi:10.1001/jama.2020.4861

58. Dong L, Tian J, He S, et al. Possible Vertical Transmission of SARS-CoV-2 From an Infected Mother to Her Newborn. JAMA.

2020;323(18):1846-1848. doi:10.1001/jama.2020.4621

59. Yan J, Guo J, Fan C, et al. Coronavirus disease 2019 in pregnant women: A report based on 116 cases. Am J Obs Gynecol MFM. 2020;223(1):111.e1-111.e14. doi:10.1016/j.ajog.202 $\underline{0.04 .014}$

60. Xiong Y, Zhang Q, Zhao L, Shao J, Zhu W. Clinical and Imaging Features of COVID-19 in a Neonate. Chest. 2020;158(1):e5-e7. doi:10.1016/j.chest.2020.0 $\underline{3.018}$

61. Duran P, Berman S, Niermeyer S, et al. COVID-19 and newborn health: Systematic review. Pan Am J Public Heal. 2020;44:1-12. doi:10.26633/rpsp.2020.54

62. World Health Organisation. Clinical management of severe acute respiratory infection (SARI) when COVID-19 disease is suspected. Interim guidance. Pediatr Med Rodz. 2020;16(1):9-26. doi:10.15557/pim $\underline{\text { r.2020.0003 }}$

63. de Souza TH, Nadal JA, Nogueira RJ, Pereira RM, Brandao MB. Clinical Manifestations of Children with COVID-19. a Systematic Review medRxiv. 2020. doi:1 0.1002/ppul.24885.doi:

64. Dong Y, Mo X, Hu Y, et al. Epidemiology of COVID-19 Among Children in China. Pediatrics. 2020;145(6):e20200702. doi:10.1542/peds.2020-0702 
65. Wang D, Hu B, Hu C, et al. Clinical Characteristics of 138 Hospitalized Patients With 2019 Novel Coronavirus-Infected Pneumonia in Wuhan, China. JAMA. 2020;323(11):1061-1069. doi:10.1001/jama.20 20.1585

66. Chen N, Zhou M, Dong X, et al. Epidemiological and clinical characteristics of 99 cases of 2019 novel coronavirus pneumonia in Wuhan, China: A descriptive study. The Lancet.

2020;395(10223):507-513. doi:10.1016/s0140-6736(2 0)30211-7

67. Shekerdemian LS, Mahmood NR, Wolfe KK, et al. Characteristics and Outcomes of Children With Coronavirus Disease 2019 (COVID-19) Infection Admitted to US and Canadian Pediatric Intensive Care Units. JAMA Pediatr. 2020;174(9):868. doi:10.10 01/jamapediatrics.2020.1948

68. Guan W, Ni Z, Hu Y, et al. Clinical Characteristics of Coronavirus Disease 2019 in China. N Engl J Med. 2020;382(18):1708-1720. doi:10.1056/nejmoa2002032

69. Huang C, Wang Y, Li X, et al. Clinical features of patients infected with 2019 novel coronavirus in Wuhan, China. The Lancet. 2020;395(10223):497-506. doi:10.1016/s0140-6736(20)30183-5

70. Tan L, Wang Q, Zhang D, et al. Lymphopenia predicts disease severity of COVID-19: A descriptive and predictive study. Sig Tranduct Target Ther. 2020;5:16-18.
71. Parri N, Lenge M, Buonsenso D. Children with Covid-19 in Pediatric Emergency Departments in Italy. N Engl J Med. 2020;383(2):187-190. doi:10.105 6/nejmc2007617

72. Xiao F, Tang M, Zheng X, Liu Y, Li X, Shan H. Evidence for Gastrointestinal Infection of SARSCoV-2. Gastroenterology. 2020;158(6):1831-1833.e3. doi:10.1053/j.gastro.2020.02.055

73. Liu J, Cao R, Xu M, et al. Hydroxychloroquine, a less toxic derivative of chloroquine, is effective in inhibiting SARS-CoV-2 infection in vitro. Cell Discov. 2020;6(1):16. doi:10.1038/s41421-020-0156-0

74. Lagier J-C, Million M, Gautret P, et al. Outcomes of 3,737 COVID-19 patients treated with hydroxychloroquine/azithromycin and other regimens in Marseille, France: A retrospective analysis. Travel Med Infect Di. 2020;36:101791. doi:1 0.1016/j.tmaid.2020.101791

75. Mitja O, Corbacho-monne M, Ubals M, et al. Hydroxychloroquine for early treatment of adults with mild covid-19: A randomized controlled trial. Clin Infec Dis. 2020. doi:10.1093/cid/ciaa1009

76. FDA. Coronavirus ( COVID-19) update: FDA issues emergency us authorization for potential COVID-19 treatment. 2020. https://www.fda.gov/news-events/pr ess-announcements/coronavirus-covid-19-update-fd a-issues-emergency-use-authorization-potential-covi d-19-treatment. Accessed August 20, 2020. 


\section{SUPPLEMENTARY MATERIALS}

\section{Online Supplementary Document}

Download: https://www.joghr.org/article/16684-neonatal-covid-19-exposures-and-infections-a-systematic-review-ofmodes-of-transmission-manifestations-and-management/attachment/44999.pdf 